\title{
The Effects of the Galactic Magnetic Field on UHECR From Local Sources
}

\section{Andrew M. Taylor*}

Deutsches Elektronen-Synchrotron (DESY), Platanenallee 6, D-15738 Zeuthen, Germany

E-mail: andrew.taylor@desy.de

\section{A. M. Hillas ${ }^{\dagger}$}

School of Physics and Astronomy, University of Leeds, Leeds LS2 9JT, UK

\begin{abstract}
We summarise the results found following a study of the effects that Galactic magnetic fields can have on the propagation of cosmic rays from local extragalactic sources. This study focuses on the coherent structures in the Jansson-Ferrar Galactic magnetic field model, namely the: disk field, a toroidal field, and an x-field components. The phenomena of Galactic magnetic field shadowing, source deflection, and Galactic magnetic field tunnel vision are all noted. Attention throughout this study is placed on particles with rigidity around $10^{18.5} \mathrm{EV}$, believed to dominate the cosmic ray spectrum above the ankle. The Galactic magnetic field component predominantly responsible for giving rise to each of these different effects was determined.
\end{abstract}

36th International Cosmic Ray Conference -ICRC2019-

July 24th - August 1st, 2019

Madison, WI, U.S.A.

\footnotetext{
* Speaker.
}

${ }^{\dagger}$ Deceased. 


\section{Introduction}

Although the origin of UHECR still remains unknown, a wealth of information continues to mount about their candidate sources.

The maximum energy for a nuclei achievable by acceleration within a candidate source [1], can be estimated through a balance of the acceleration timescale with either the source activity or escape timescales. By expressing the acceleration timescale $t_{\text {acc }}$ as a multiple $\eta$ of the Larmor time $R_{\mathrm{L}} / c=p /(e B)$ of a particle of momentum $p$ around the magnetic field (strength $B$ ): $t_{\text {acc }}=\eta R_{\mathrm{L}} / c$. For a source's maximum energy to reach up to UHECR energies, it must operate as an extreme accelerator, achieving close to Bohm level scattering times, with $\eta \approx 1$.

A tight lower bound can be placed on the magnetic luminosity of an UHECR sources, [3], which can be written as $L_{B} \gtrsim 10^{43} \eta^{2}\left(R_{\max } / 10^{19} \mathrm{~V}\right)^{2} \mathrm{erg} / \mathrm{s}$, where $R=E / Z$ is the rigidity of the UHECRs in the observer frame. A maximum rigidity of $10^{19} \mathrm{~V}$, that is $E=10^{19} \mathrm{eV}$ for protons $(Z=1)$ or $E \sim 10^{20} \mathrm{eV}$ for Silicon $(Z=14)$, is in line with the current best constraints from the Pierre Auger Observatory [4, 5].

The propagation of UHECR is limited by their interaction with extragalactic background radiation fields. A maximum distance scale of $80 \mathrm{Mpc}$ has been previously determined, within which the nearest by UHECR sources must exist [2]. Although no blazars sit within this range, several examples of their misaligned counterparts (radiogalaxies) do satisfy this local proximity constraint.

As an example of such objects, local radiogalaxy such as Cen A exist, whose jet power appears to sufficient in order reach to UHECR energies. The jet power of Cen A being of the order of $10^{43} \mathrm{erg} / \mathrm{s}$ [6], the above estimates suggest that this local misaligned AGN, and others like it, are capable of accelerating nuclei up to the UHECR energy scale, provided $\eta$ is as low as unity at the highest energies. Hence, whether blazars and radio galaxies can actually contribute to the UHECR spectrum or not, depends both on how efficient the acceleration can be, and on the composition of UHECRs themselves.

Of the deflection of UHECR during their propagation in the extragalactic environment, the deflection of UHECR from local extragalactic sources will be smallest due to their reduced residence time within extragalactic magnetic fields. The deflection of UHECR within the Galactic magnetic field can be of particular relevance for particles originating from local sources. Likewise, a sufficiently accurate understanding of the Galactic magnetic field structure allows the promise a recovery of the UHECR flux impinging on the Galactic magnetosphere, whose anisotropy may be dominated by the local source fluxes.

We here focus on obtaining an understanding of the effects that the Galactic magnetic field can have on the arriving UHECR. By further considering the components of the Galactic magnetic field presently considered in a popular Galactic magnetic field model, the field components predominantly giving rise to these effects are isolated.

\section{The Galactic Magnetic Field Model}

The Galactic magnetic field model considered here is the regular component of the JF12 model [7]. This consists of three separate structured field components, namely: a disk field component 
(disk), a toroidal halo component (toroidal), and an $\mathrm{x}$-field component (x-field). The field resulting from the sum of these components we refer to throughout as total.

One of the constraints on this proposed Galactic magnetic field structure have been provided by Faraday rotation measure measurements from an ensemble of region in the sky. Such observations constrain the parallel component of the magnetic field to the line-of-sight, requiring also the adoption of a Galactic thermal electron distribution in order to provide such a constraint.

Polarised synchrotron emission maps can provides a complimentary constraint on the Galactic magnetic field, providing input on the perpendicular component of the field to the line-of-sight. The use of synchrotron maps to provide such a constraint, however, first require the adoption of a Galactic non-thermal electron distribution.

The inference of the Galactic magnetic field structure is therefore limited by both our incomplete knowledge of the thermal and non-thermal contents of the Galaxy, as well as current observational limitations in our ability to probe Galactic rotation measure and synchrotron emission.

\section{The Influence of the Galactic Magnetic Field on UHECR}

It is also interesting to consider the energy content of the three Galactic magnetic field components of the JF12 model. These are $U_{\mathrm{B}}^{\text {disk }}=8 \times 10^{53} \mathrm{erg}, U_{\mathrm{B}}^{\text {toroid }}=4 \times 10^{54} \mathrm{erg}$, and $U_{\mathrm{B}}^{\mathrm{x}-\text { field }}=$ $3 \times 10^{54} \mathrm{erg}$. In comparison, the Galactic CR population is estimated to maintain a total energy content of $U_{\mathrm{CR}}=3 \times 10^{55} \mathrm{erg}$ [8]. Furthermore, a consideration of the spatial distribution of these magnetic field components, suggests that only the toroidal and $\mathbf{x}$-field dominate the out-of-plane Galactic magnetic field structure, and therefore are of most relevance for the majority of extragalactic source.

With the Galactic magnetic field structured components considered possessing sizes larger than the Larmor radii of $3 \times 10^{18} \mathrm{eV}$ protons in $\mu G$ strength magnetic fields, the presence of these structures is naturally expected to distort the flux passing through the Galaxy. Specifically, both the spatial distribution of the cosmic rays throughout the Galaxy, and their angular distribution locally at a point within in, will be collectively effected.

\subsection{Shadowing}

We here consider a setup in which UHECR from Cen A (with Galactic coordinates $l=309.5^{\circ}$, $b=19.4^{\circ}$ ) arrive to the Galactic magnetosphere in a parallel beam of radius $30 \mathrm{kpc}$. With an injection distance from the Galactic center for this beam of $30 \mathrm{kpc}$ being adopted, half of the Galactic magnetosphere was illuminated by the beam.

Following the injection of these $10^{18.5} \mathrm{eV}$ protons into the system, their subsequent trajectories were tracked until their eventual escape. A Galactic escape boundary of $30 \mathrm{kpc}$ was here adopted. Following the assumption that the source is continuously illuminating the Galactic magnetosphere, the steady-state density in the Galactic disk region (within $100 \mathrm{pc}$ from the plane) was determined.

The results for the case of only single component of the Galactic magnetic field, namely $\mathbf{x}$ field, toroidal, and disk are shown in fig. 1. Both components whose contribution outside the Galactic disk plane is significant (the $\mathbf{x}$-field and toroidal components) are found to lead to a considerable level of shadowing of the region on the far side of the Galactic center relative to the 

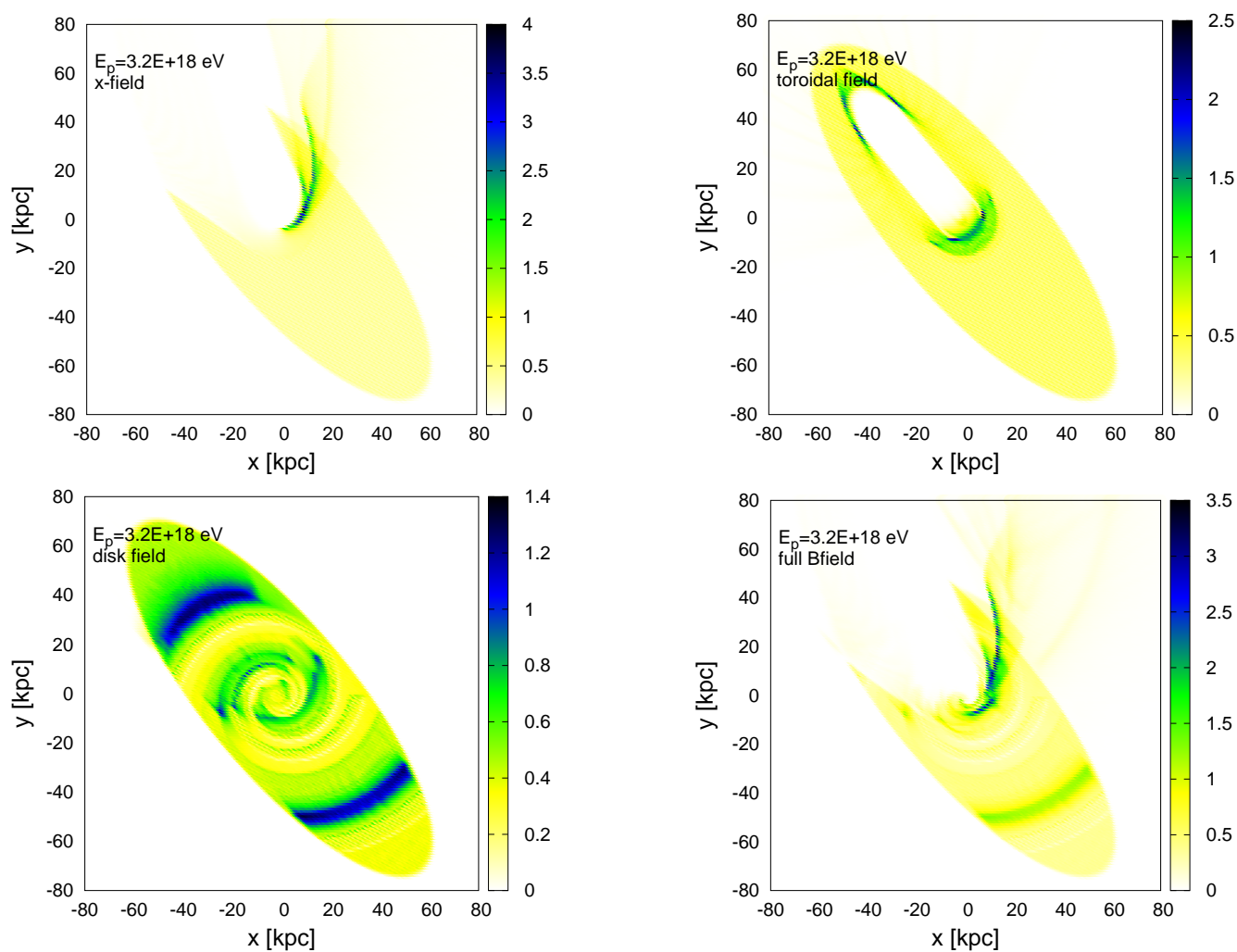

Figure 1: The steady-state $\mathrm{CR}$ density in the Galactic disk region following the continuous injection of $10^{18.5} \mathrm{eV}$ protons (see injection setup description in the main text). The top-left/top-right/bottomleft/bottom-right plots shows the result for the $\mathbf{x}$-field/toroidal/disk/total Galactic magnetic field components.

source position. It should be noted that the overall elliptical illumination shape within the Galactic plane results from the projection effect of the inclined source beam onto the Galactic plane surface.

The presence of such a Galactic shadowing process is somewhat analogous to [9], where the dense magnetic field surrounding the Sun leads to shielding of the Galactic cosmic rays from a region around the Solar disk. However, in the case considered here, the shadowing process is instead be being applied to extragalactic CR from a local source, following their passing close the Galactic center region.

\subsection{Shifting in Cen A Source Position}

Utilising the same setup for the propagation of CR as described in the previous section, the anisotropy of the particles arriving to the Earth location was next considered. Although the particle beam recalled its general origin, a degree of shifting of the beam position relative to its initial direction was found (see bottom-right panel in fig. 2).

This overall shift in the source position is consistent with the finding in [10]. It should be noted that although we focus here on the injection of particles from the source direction, the method of backtracking particles from Earth's position was also found to give consistent results. 

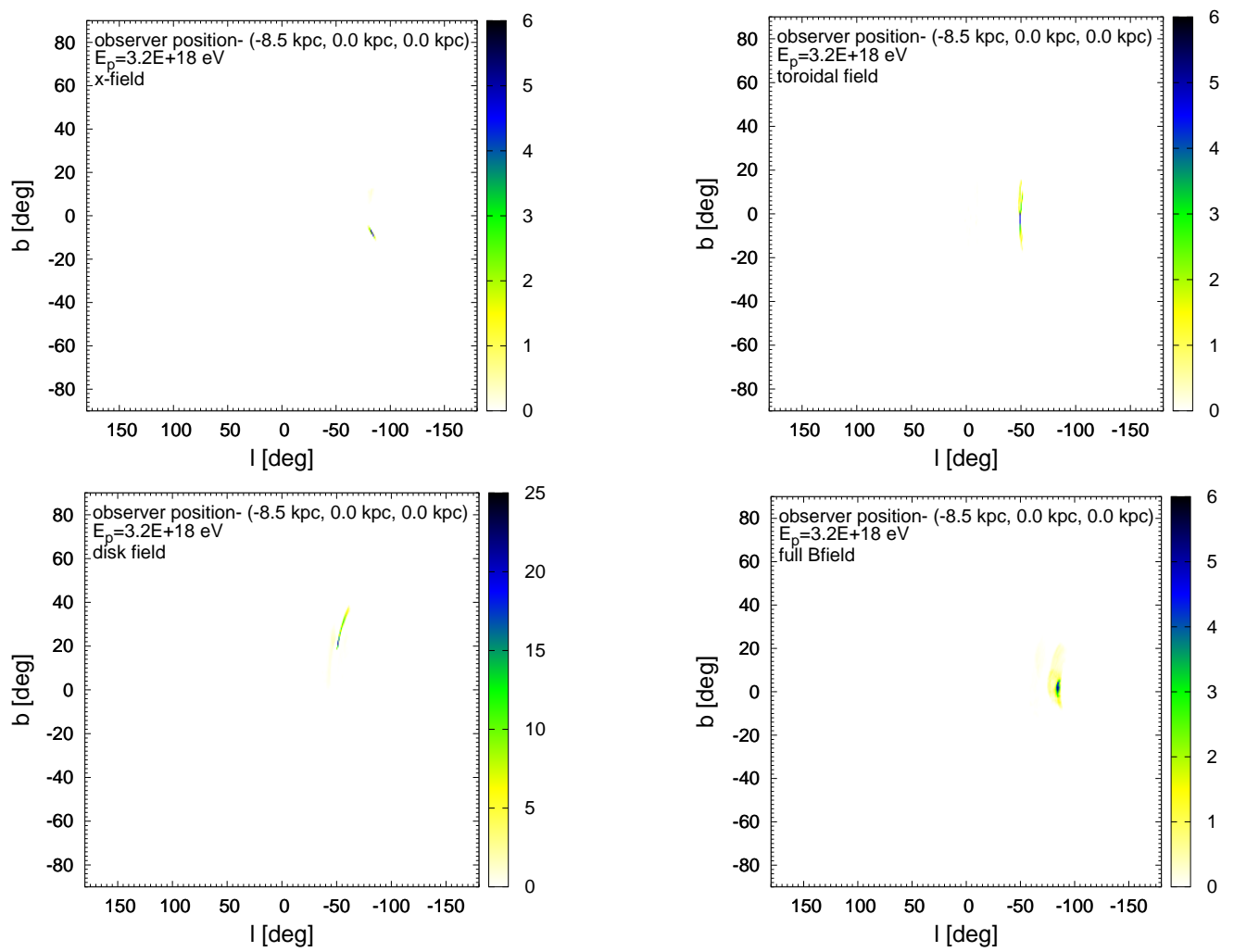

Figure 2: The arriving CR anisotropy skymaps following the injection of $10^{18.5} \mathrm{eV}$ protons (see injection setup description in the main text). The top-left/top-right/bottom-left/bottom-right plots shows the result for the $\mathbf{x}$-field/toroidal/disk/total Galactic magnetic field components.

An investigation was next carried out in order to determine which component of the Galactic magnetic field played the dominant role in the shifting of the source position. This was carried out by a switching off of the disk, torus, and $\mathbf{x}$-field components. The findings are shown in the top-left, top-right, and bottom-left panels in fig. 2. It is evident from these results that the $\mathbf{x}$ field component dominated the coherent shifting of the source position, particularly in the Galactic longitude. The removal of this field component being found to allow a much broader angular distribution range of $\mathrm{CR}$ to arrive from Cen $\mathrm{A}$.

\subsection{Tunnel Vision}

Finally, the part of the extragalactic sky that our present location within the Galaxy forces us to preferentially observe was probed. Starting with an isotropic ensemble of $10^{18.5} \mathrm{eV}$ protons from Earth and backtracking them through the Galactic magnetic field, their subsequent anisotropic probing of the extragalactic sky was investigated. The results for the backtracking through the different individual components of the Galactic magnetic field are shown in fig. 3.

It is apparent from these results that the Northern extragalactic sky (ie. positive Galactic latitudes) is preferentially probed in direction at angles point from the Galactic center direction. The origin for this preference can be understood from the toroid plot in fig. 3. This figure demonstrates the toroid Galactic magnetic field component acts as a magnetic lenses, deterring flux from south- 

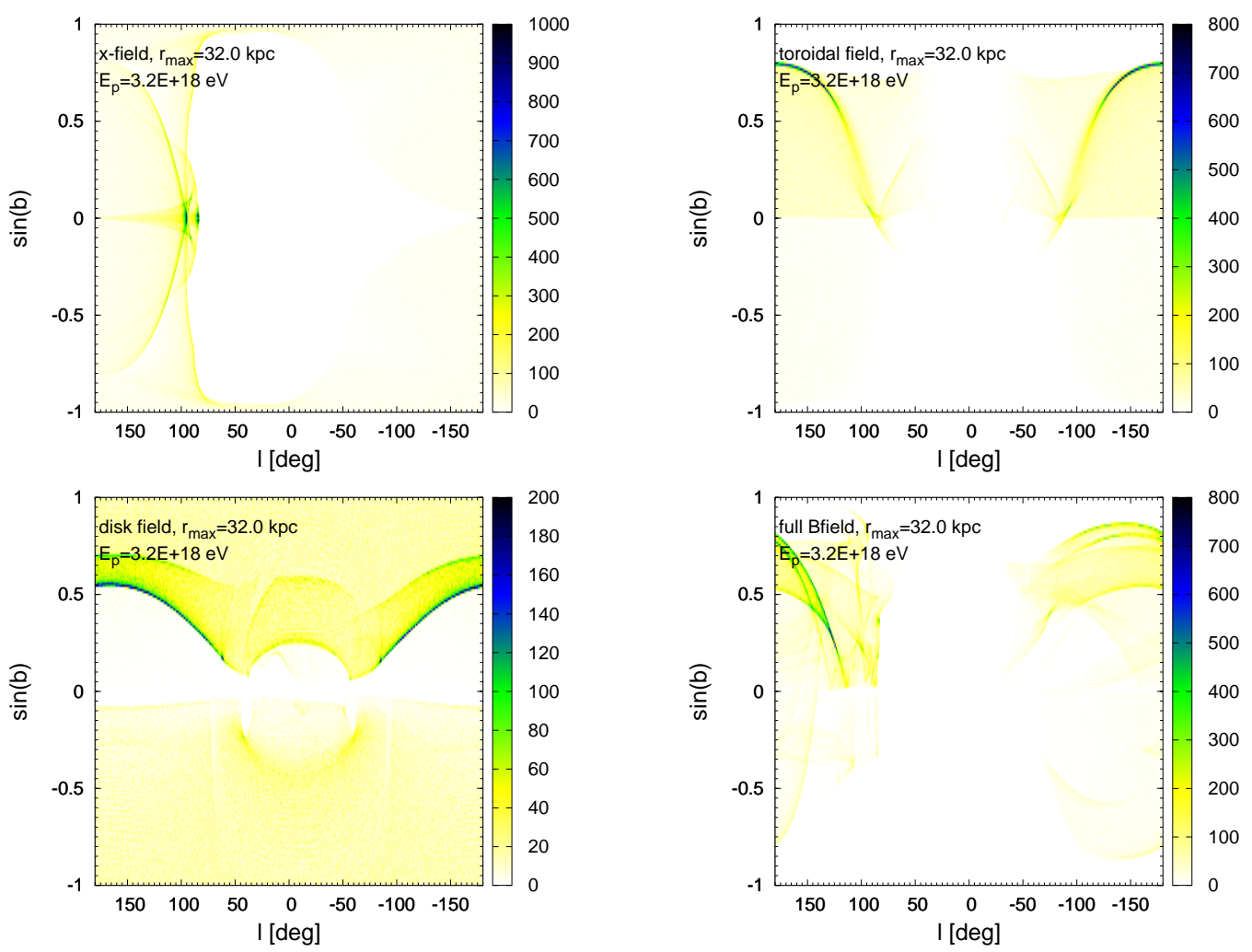

Figure 3: Skymaps of the backtracked CRs following the injection of isotropic distribution of $10^{18.5} \mathrm{eV}$ protons at Earth. The top-left/top-right/bottom-left/bottom-right plots shows the result for the $\mathbf{x}$ field/toroidal/disk/total Galactic magnetic field components.

ern Galactic latitudes. The origin of the preferential sampling directions at large angles to that of the Galactic center region is instead found to originate from the presence of both toroid and $\mathbf{x}$-field components.

\section{Conclusion}

Motivated by the consideration of local radiogalaxies as UHECR sources, the effect of the potential coherent Galactic magnetic field structures on their entry into the Galactic magnetosphere are considered.

Shadowing of $10^{18.5} \mathrm{eV}$ UHECR passing close to the Galactic center region for trajectories originating from Cen A was found. This result was noted to be influenced by both the toroidal and $\mathbf{x}$-field Galactic magnetic field components, with the latter of the two components dominating most severely this shadowing effect.

For $10^{18.5} \mathrm{eV}$ UHECR originating from the direction of Cen A, a shifting of the source direction was also seen. This shifting was again found to be influenced by the toroidal and $\mathbf{x}$-field Galactic magnetic field components, with the latter of the two having the most coherent influence on the source shifting. 
Lastly, the tunnel vision due to our observation of Galactic UHECR from Earth's location within the Galactic magnetic field was considered. The parts of the extragalactic sky preferentially probed by UHECR observation at Earth was considered by backtracking of an isotropic distribution of UHECR at Earth through the Galactic magnetic field.

\section{References}

[1] A. M. Hillas, Ann. Rev. Astron. Astrophys. 22, 425 (1984).

[2] A. M. Taylor, M. Ahlers and F. A. Aharonian, Phys. Rev. D 84 (2011) 105007 doi:10.1103/PhysRevD.84.105007 [arXiv:1107.2055 [astro-ph.HE]].

[3] C. A. Norman, D. B. Melrose, A. Achterberg, Astrophysical Journal 454 60N (1995)

[4] A. Aab et al. [Pierre Auger Collaboration], Phys. Rev. D 90 (2014) no.12, 122006 doi:10.1103/PhysRevD.90.122006 [arXiv:1409.5083 [astro-ph.HE]].

[5] R. Alves Batista et al., Front. Astron. Space Sci. 6 (2019) 23 doi:10.3389/fspas.2019.00023 [arXiv:1903.06714 [astro-ph.HE]].

[6] S. Wykes et al., Astron. Astrophys. 558 (2013) A19 doi:10.1051/0004-6361/201321622 [arXiv:1305.2761 [astro-ph.HE]].

[7] R. Jansson and G. R. Farrar, Astrophys. J. 757 (2012) 14 doi:10.1088/0004-637X/757/1/14 [arXiv:1204.3662 [astro-ph.GA]].

[8] L. O. Drury, Astropart. Phys. 39-40 (2012) 52 doi:10.1016/j.astropartphys.2012.02.006 [arXiv:1203.3681 [astro-ph.HE]].

[9] M. Amenomori et al. [Tibet ASgamma Collaboration], Phys. Rev. Lett. 111 (2013) no.1, 011101 doi:10.1103/PhysRevLett.111.011101 [arXiv:1306.3009 [astro-ph.SR]].

[10] A. Keivani, G. R. Farrar and M. Sutherland, Astropart. Phys. 61 (2014) 47 doi:10.1016/j.astropartphys.2014.07.001 [arXiv:1406.5249 [astro-ph.HE]]. 